\title{
The value of embedded secondary-care-based psychology services in rheumatology: an exemplar for long-term conditions
}

\author{
Authors: Theresa Barnes, ${ }^{A}$ Lou Taylor, ${ }^{B}$ Charlotte Eost-Telling ${ }^{B}$ and Thomas Joy ${ }^{C}$
}

\section{Introduction}

Rheumatoid arthritis is an exemplar long term condition, complicated by pain, disability, comorbidities and long-term medication use. It has significant effects on mobility, work performance, social role, sexual function and relationships. It is commonly associated with fatigue and mood disturbance as a result of complex interactions of physical (disease related) and psychosocial factors. ${ }^{1}$ National Institute for Health and Care Excellence guidance recommends the availability of psychological support for these patients. ${ }^{2}$ We have implemented a psychology service for our patients with chronic rheumatological conditions. This study was set up to capture the value of this service.

\section{Materials and methods}

Patients were assessed before and after psychology intervention using validated questionnaires: HAQ (disability), HADS (anxiety and depression), and a measure of patient self-efficacy. Structured interviews were used to analyse the value of the service to individual service users and analysed for themes. Finally, social return on investment (SROI) methodology was used to assess the monetary value of the service. ${ }^{3}$ SROI is a framework for measuring and accounting for the value created or destroyed by our activities - where the concept of value is much broader than that which can be captured by market prices. SROI seeks to reduce inequality and environmental degradation and improve wellbeing by taking account of this broader value.

\section{Results}

Results demonstrate no change in HAQ scores, an improvement in HADS (anxiety and depression) and an improvement in self-efficacy at the end of treatment (Fig 1).

Structured interviews identified the following themes; the service gave patients permission to tell family and friends about the impact of the disease; it gave patients 'tools' to aid management; it provided another perspective for exploring the disease; and

Authors: ${ }^{A}$ Countess of Chester Hospital, Chester, UK; ${ }^{B}$ University of Chester, Chester, UK; ${ }^{C}$ Cheshire and Wirral Partnership, Chester, UK

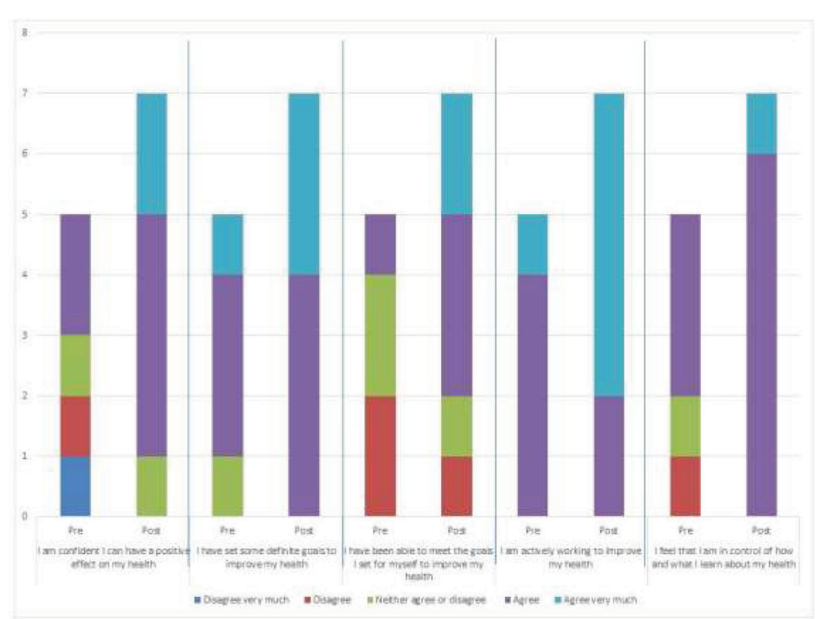

Fig 1. Patient activation domains pre- and post-psychological intervention.

it enabled patients to be able to pull together all aspects of the disease. SROI demonstrated that the service was highly valued by the patients.

\section{Conclusions}

Psychological support was highly valuable to our patients with chronic rheumatological disease as demonstrated by SROI. It led to an improvement in anxiety and depression. Studies have demonstrated that higher levels of depression are associated with an increase in pain perception and disease activity assessments. ${ }^{4}$ Psychology input did not lead to an improvement in disability but did lead to an improvement in patient selfefficacy which it is anticipated will lead to a decrease in health resource utilisation. ${ }^{5}$

\section{Conflicts of interest}

None declared. 


\section{References}

1 Nikolaus S, Bode C, Taal E, van de Laar M. Fatigue and factors related to fatigue in rheumatoid arthritis: a systematic review. Arthritis Care Res 2013:65:1128-46.

2 National Institute for Health and Care Excellence. Rheumatoid arthritis in adults: management: NICE guideline [NG100]. NICE, 2018. www.nice.org.uk/guidance/ng100

3 Cordes J]. Using cost-benefit analysis and social return on investment to evaluate the impact of social enterprise: Promises, implementation, and limitations. Eval Program Plann 2017;64:98-104.
4 Matcham F, Norton S, Scott DL, Steer S, Hotopf MJR. Symptoms of depression and anxiety predict treatment response and long-term physical health outcomes in rheumatoid arthritis: secondary analysis of a randomized controlled trial. Rheumatology 2015;55:68-278.

5 De Silva D. Evidence: Helping people help themselves. London: The Health Foundation; 2011. 